\title{
OPERATION AND CONTROL OF SPLIT-PARALLEL, THROUGH-THE- ROAD HYBRID ELECTRIC VEHICLE WITH IN-WHEEL MOTORS
}

\author{
Saiful A. Zulkifli ${ }^{1,3 *}$, Syaifuddin Mohd ${ }^{2,3}$, Nordin Saad ${ }^{1}$ and A. Rashid A. Aziz ${ }^{2,3}$ \\ ${ }^{1}$ Electrical and Electronic Engineering Department \\ ${ }^{2}$ Mechanical Engineering Department \\ ${ }^{3}$ Centre for Automotive Research and Electric Mobility (CAREM) \\ Universiti Teknologi PETRONAS, 32610 Bandar Seri Iskandar, Perak, Malaysia \\ Email: saifulazrin_mz@petronas.com.my \\ Phone: +605 368 7852; Fax: +6053657443
}

\begin{abstract}
A variant of the hybrid electric vehicle (HEV) is a split-axle, through-the-road parallel hybrid, which enables existing vehicles to be retrofitted into an HEV with minimal modification - achieved by placing electric motors in the hub of the otherwise non-driven wheels. The term 'through-the-road' (TTR) refers to the power coupling scheme between the internal combustion engine (ICE) and in-wheel motors (IWM), which is not through some mechanical device but through the vehicle itself, its wheels and the road on which it moves. The objective of the present work is to identify specific operating modes, power flow, torque-speed relationship, profiles and system architecture of the TTR-IWM hybrid - in particular a retrofit-conversion hybrid - in comparison to the ordinary parallel HEV. This paper also discusses three possible control architectures and the corresponding control parameters. The TTR-IWM hybrid's reduced operating modes and inability to charge its battery bank during vehicle stand-still present challenges which require a specific control strategy. Simulation results of acceleration show that the TTR-IWM hybrid has better fuel economy and acceleration performance, compared to the pre-transmission, parallel hybrid vehicle with on-board motor.
\end{abstract}

Keywords: HEV; split-parallel configuration; through-the-road hybrid; operation of split-parallel hybrid; control of split-parallel hybrid; in-wheel motors.

\section{INTRODUCTION}

A parallel-configuration hybrid vehicle typically has the electric motor-generator onboard the chassis, as in most production hybrids of today, but in one variant of the parallel hybrid, the electric motor is part of the wheel itself, called a hub motor, or in-wheel motor (IWM) [1, 2]. In this configuration, the ICE operates on one drive-shaft (e.g., front) while the in-wheel motors are fitted into the other pair of wheels (e.g., rear) (Figure 1), resulting in a split-axle parallel architecture [3]. There is no specific mechanical device coupling the two propulsion sources; instead, the power sources are coupled through the road, giving rise to the term through-the-road or TTR hybrid. This configuration enables an existing ICE-driven vehicle to be converted into a hybrid electric vehicle with minimal modification - by replacing the otherwise non-driven wheels with IWM and powering them with a battery bank located in the vehicle's trunk. 

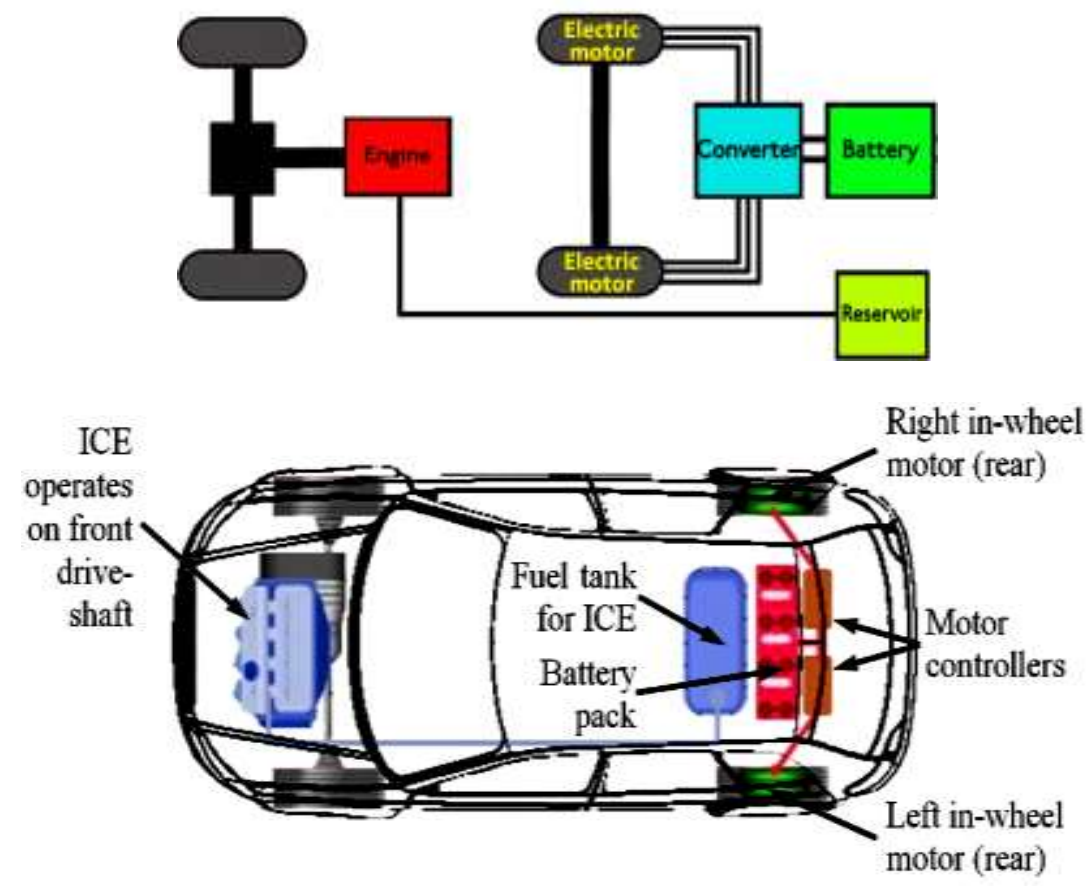

Figure 1. A split-parallel hybrid electric vehicle with in-wheel motors [3].

Along with motor controllers and an energy management system controller (EMS) that intelligently manages the vehicle's energy and power flow, they form a TTR-IWM hybrid vehicle system [4]. In the design of a new, split-parallel hybrid vehicle with on-board motor, space limitation for motor installation is not a major issue and the ICE can be sized so that its operation occurs most frequently in the high-efficiency regions. Thus, the fuel-reduction advantage of ICE downsizing can be fully explored [5, 6]. However, in the case of retrofit TTR-IWM hybrid conversion, performance benefits may be reduced due to the following factors: unchangeable size of the ICE, limited dimensioning possibility for the in-wheel motor due to space constraint of the wheel hub, and reduced operating modes and control possibility of the TTR-IWM hybrid [3, 7]. The issue of limited motor sizing is investigated in Zulkifli, Mohd [8]; the present work attempts to address the third concern: how operation and control of the TTR-IWM hybrid vehicle differ from the ordinary, device-coupled, on-board motor parallel hybrid. The objective of the present work is to describe the operation, power flow, system characteristics, control architecture, parameters and control issues of the TTR-IWM hybrid. The paper also presents simulation results of acceleration and fuel consumption performance, comparing the TTR-IWM hybrid with the pre-transmission parallel HEV.

\section{OPERATING MODES AND POWER FLOW}

\section{Operation and Power Flow of Parallel Hybrid Vehicle}

A parallel hybrid electric vehicle derives its propulsion power from two separate power trains, each having an energy source and an energy converter. The first powertrain consists of a fuel tank and a combustion engine, while the second is made up of a battery bank and an electric motor-generator. As illustrated in Figure 2, while power flow in the former is uni-directional, the latter is bi-directional - it allows for energy to be dispensed 
(electric motoring) and replenished (charging and re-generative braking). In general, this results in nine possible operating modes for a parallel HEV [3].

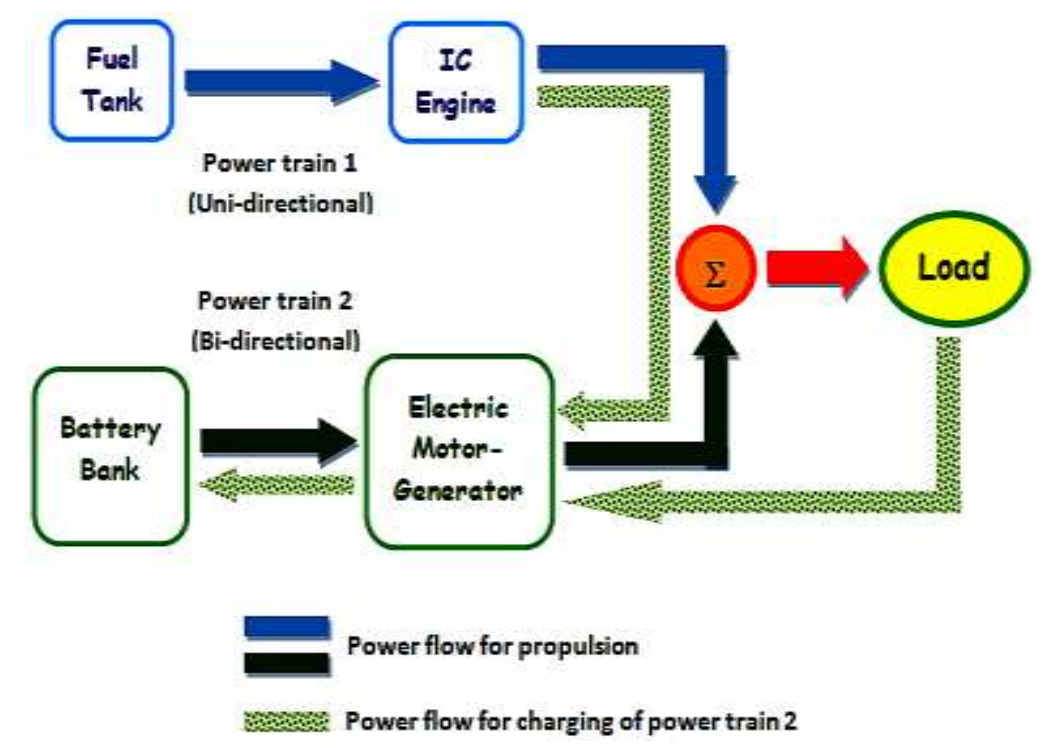

Figure 2. Power flow paths in a parallel hybrid electric vehicle.

In mode 1 , only the ICE provides propulsion power to the vehicle, while in mode 2, the electric motor alone propels the vehicle. Mode 3 has both sources providing power to the load. Mode 4 is the regenerative braking mode, while in mode 5 only the battery bank is charged directly by the engine (through the generator). The battery is simultaneously charged by the engine and through regenerative braking in mode 6 . In mode 7, the ICE charges the battery and propels the vehicle at the same time. In mode 8, the engine charges the battery while the motor propels the vehicle. In mode 9 , the engine propels the vehicle, while the battery is charged by generation from the load.

1. Powertrain 1 alone delivers power to the load.

2. Powertrain 2 alone delivers power to the load.

3. Both powertrains 1 and 2 deliver power to the load.

4. Powertrain 2 obtains power from the load.

5. Powertrain 2 obtains power from powertrain 1.

6. Powertrain 2 obtains power from powertrain 1 and the load.

7. Powertrain 1 delivers power to the load and to powertrain 2.

8. Powertrain 1 delivers its power to powertrain 2 , and powertrain 2 delivers its power to the load.

9. Powertrain 1 delivers its power to the load and the load delivers power to powertrain 2 .

\section{Operation and Power Flow of Split-Parallel TTR-IWM Hybrid Vehicle}

In a split-axle parallel hybrid vehicle with in-wheel motors, it is not possible for the ICE to directly charge the battery bank through the electric generator since the two sources are not coupled together. Rather, the batteries can be charged only when the vehicle is moving - when the wheels and in-wheel motors are turning and operating as generators. 
Charging cannot occur when the vehicle is stationary. Due to this condition, modes 5 through 8 described above cannot be attributed to a TTR-IWM hybrid: mode 8 is physically impossible while modes 5,7 and 9 do not precisely describe its operation. Mode 9 is an exclusive operation for the TTR hybrid: the ICE does not deliver power to the battery bank directly but through the load (vehicle mass) - when the vehicle is propelled by the ICE and power is delivered to the battery through the vehicle's chassis, wheels and road coupling. Figure 3(a) shows the configuration of the split-parallel hybrid vehicle, clearly showing the two power sources acting independently on different drive axles - there is no mechanical coupler as in the single-axle parallel hybrid vehicle (shown in Figures 3(b) and 3(c)). This results in only five possible operating conditions for the TTR-IWM hybrid vehicle, determined by the possible combination of energy flow paths, as depicted in Figure 4 and described below:

1. Mode A:

Similar to Mode 1, only the ICE provides propulsion power to the vehicle. The in-wheel motors are not operating.

2. Mode B:

Similar to Mode 2, only the motors provide power to propel the vehicle. The ICE is idling.

3. Mode C:

Similar to Mode 3, both the ICE and in-wheel motors provide propulsion power to the vehicle.

4. Mode D:

Similar to Mode 4, the vehicle's mechanical power is converted by the in-wheel generator to electrical power to charge the battery bank, during re-generative braking.

5. Mode E:

Similar to Mode 9, while the ICE propels the vehicle, the in-wheel generator converts the vehicle's energy to charge the battery bank.

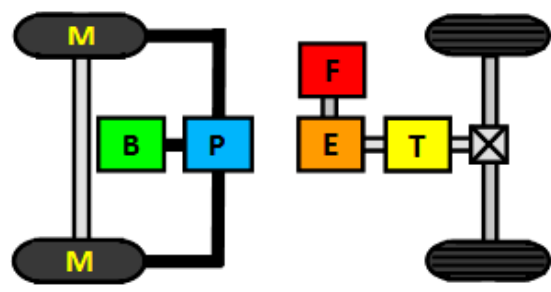

(a)

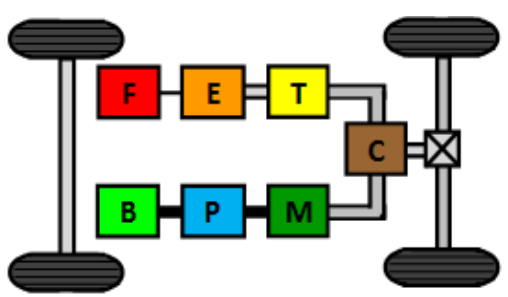

(b)
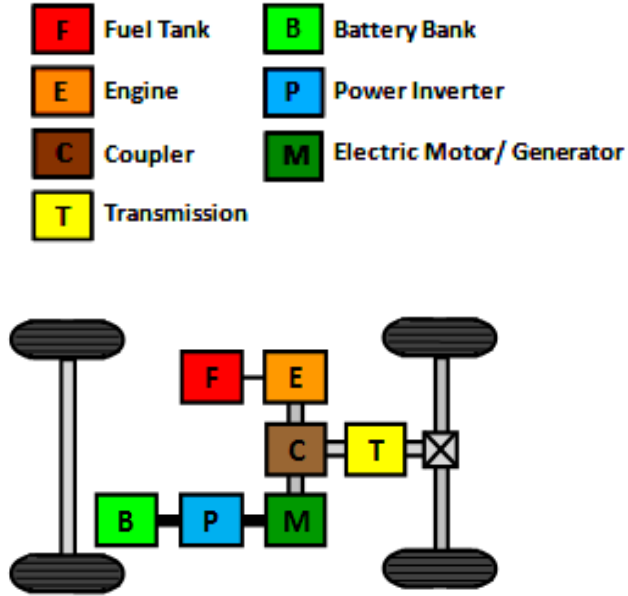

(c)

Figure 3. Drivetrain configuration: (a) TTR-IWM split-parallel hybrid;

(b) single-axle, post-transmission coupled parallel hybrid; (c) single-axle, pre-transmission coupled parallel hybrid. 

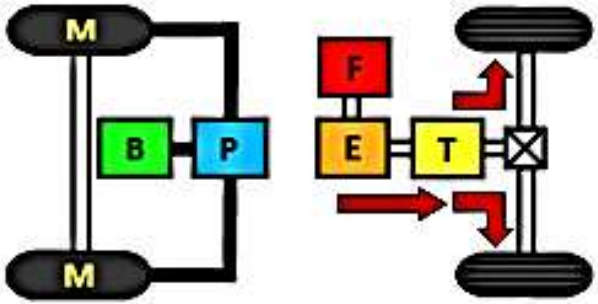

Mode A: ICE propulsion only
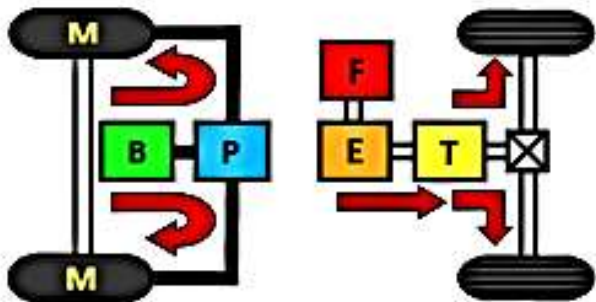

Mode C: ICE + motor propulsion
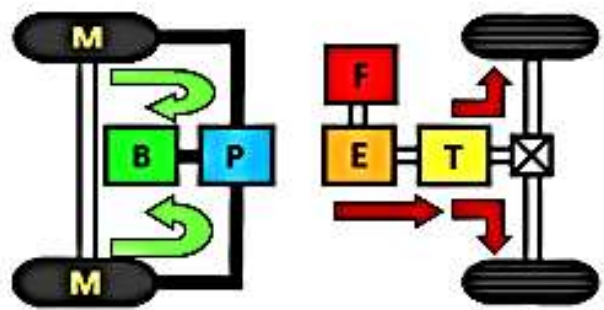

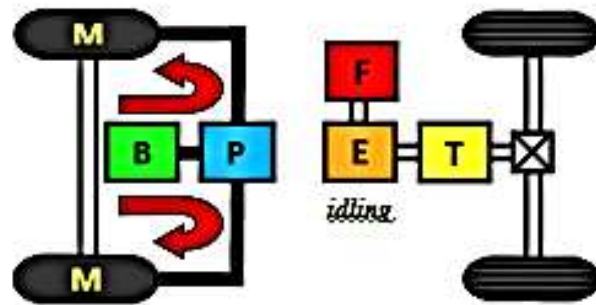

Mode B: Motor propulsion only
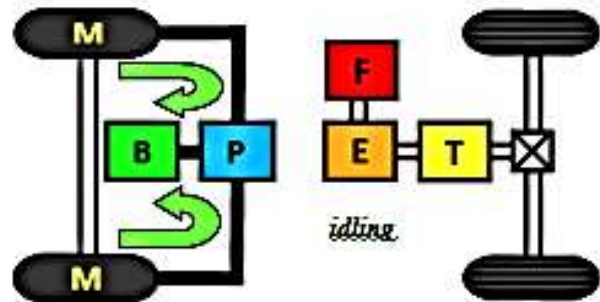

Mode D: Re-generative braking (battery charging only)

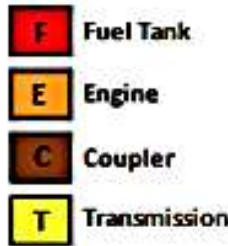

Mode E: ICE propulsion + battery charging

Figure 4. Five operating modes of split-parallel TTR-IWM hybrid vehicle.

\section{TORQUE-SPEED RELATIONSHIP, PROFILE AND SYSTEM ARCHITECTURE}

\section{Torque-Speed Relationship and Profile of TTR-IWM Hybrid}

A parallel hybrid vehicle is essentially a torque-coupled system consisting of a mechanically-coupled drivetrain. An ordinary parallel hybrid has some form of coupling device such as a pulley, gear or chain assembly, which adds together mechanical powers from the two sources. Figure 5 shows a parallel hybrid drivetrain represented by a 3-port, 2-degrees-of-freedom mechanical system. Port 1 is a uni-directional input (connected to the ICE), while port 2 (connected to the motor) and port 3 (connected to the wheels) are bi-directional input or output of the system (at least one must be an output) [5].

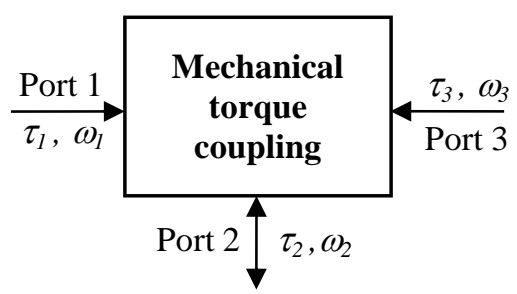

Figure 5. Three-port representation of a torque-coupled parallel hybrid drivetrain. 
Ports 1 and 2 can be connected either directly to the power sources or through a mechanical transmission. Ignoring losses, power input to the torque coupler is equal to its power output, as expressed below in Eq. (1):

$$
\tau_{3} \omega_{3}=\tau_{1} \omega_{1}+\tau_{2} \omega_{2}
$$

where $\tau_{1}$ and $\omega_{1}$ are torque and speed input of the ICE, $\tau_{2}$ and $\omega_{2}$ are torque and speed input of the electric motor, and $\tau_{3}$ and $\omega_{3}$ are torque and speed output of the torque coupler, which is connected to the final drive and wheels.

Torque output $\tau_{3}$ can be expressed as:

$$
\tau_{3}=k_{1} \tau_{1}+k_{2} \tau_{2}
$$

where $k_{1}$ and $k_{2}$ are structural parameters of the coupling system, described for example by gear ratios, and are thus fixed parameters of the system. The contributing torques $\tau_{1}$ and $\tau_{2}$ are independent of each other and can thus be individually controlled. However, due to power conservation, Eq. (1) leads to the constraint that the speeds of the power sources are linked together and governed by the relationship:

$$
\omega_{3}=\frac{\omega_{1}}{k_{1}}=\frac{\omega_{2}}{k_{2}}
$$

Thus, the sources' speeds cannot be individually controlled. Coupling of the sources can consist of either single-shaft or double-shaft configuration. The former has the motor and engine connected in-line on the same shaft, while the latter has two separate shafts connected via the gear or chain assembly. The split-axle, TTR parallel hybrid is a torque-coupled configuration; however, torque from the ICE and electric motor are added up not through a coupling device, but through the vehicle chassis, wheels and road. It is topologically similar to the dual-shaft parallel configuration, since the motor and ICE operate on separate drive axles. The torques can be individually controlled but their speeds are governed by Eq.. In the case of a retrofit-conversion TTR-IWM vehicle, the ICE is connected to a multi-gear transmission (existing) while there is no transmission for the motor since it is in the wheel hub (similar to a directly-coupled on-board motor). Hence, with port 3 connected to the front wheels and port 1 connected to the ICE, $k_{1}$ is determined by the gear position of the ICE's transmission. Port 2 is connected to the motor with $k_{2}=1$ (direct-coupled, in-wheel motor). Thus, Eq. (2) and Eq. become:

$$
\begin{aligned}
& \tau_{3}=k_{1} \tau_{1}+\tau_{2} \\
& \omega_{3}=\frac{\omega_{1}}{k_{1}}=\omega_{2} .
\end{aligned}
$$

The in-wheel motor speed $\omega_{2}$ is thus equal to the speed of the ICE-driven front wheels, $\omega_{3}$. This is similar to a double-shaft, device-coupled parallel drivetrain with the motor shaft coupled directly to the front-wheel axle (post-transmission coupling) with $k=1$. In a torque-coupled hybrid vehicle, the torque from both sources combines to give a final traction profile. The resultant tractive profiles of a TTR-IWM hybrid vehicle are shown in Figure 6(a). As mentioned above, its traction profiles are similar to a 
device-coupled parallel hybrid vehicle with single-axle, dual-shaft, post-transmission coupling, single-gear motor and multi-geared ICE, as shown in Figure 6(b).

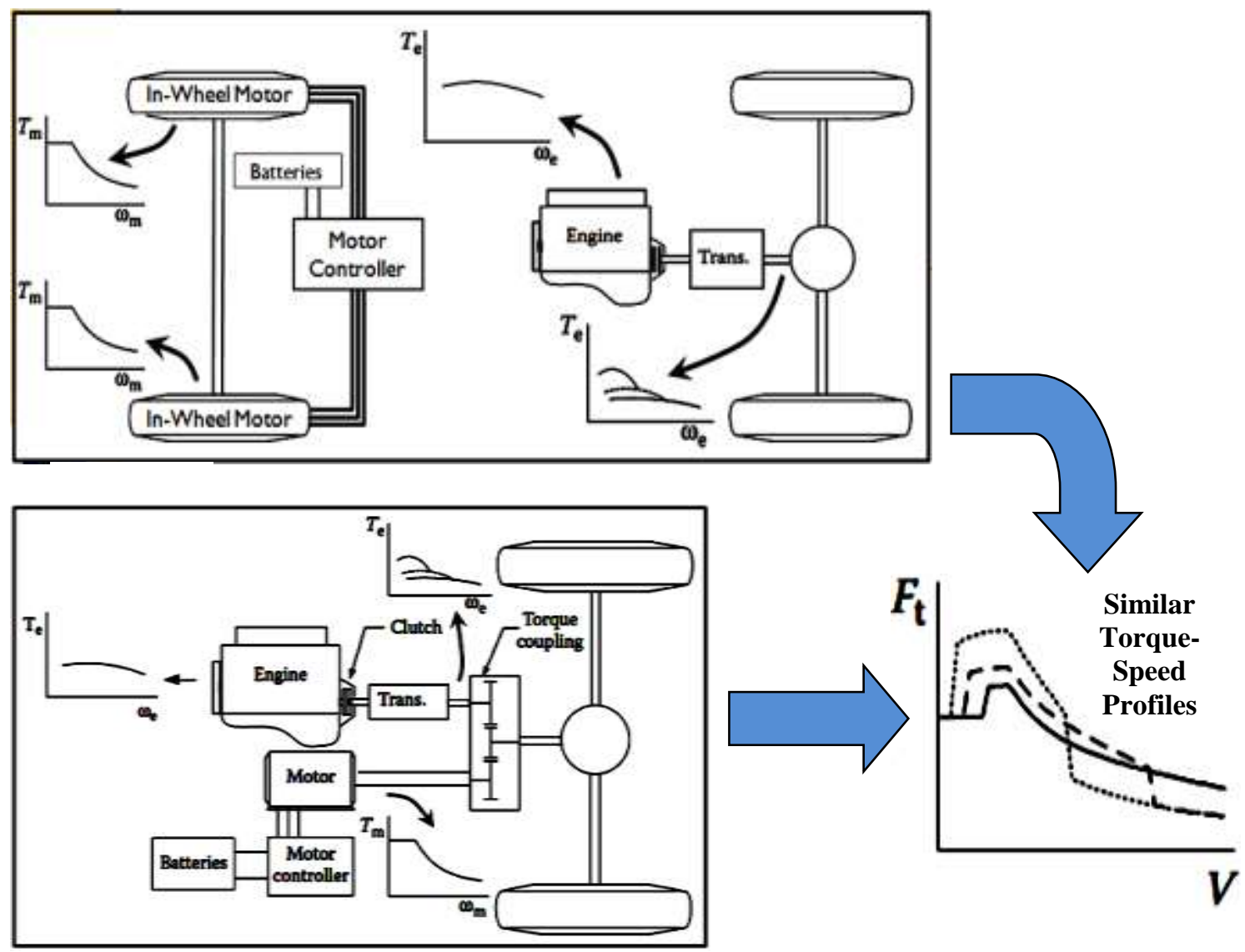

Figure 6. Resultant torque-speed profiles: (a) TTR-IWM hybrid; (b) single-axle, parallel hybrid with post-transmission, single-gear motor and multi-geared ICE.

\section{System Architecture of TTR-IWM Hybrid}

Figure 7 shows the system architecture of a split-parallel TTR-IWM hybrid, applicable to both purpose-built and retrofit-conversion vehicles. At the heart of the system is the main controller, referred to as the energy management system (EMS). The EMS is connected to both the engine control unit (ECU) and the motor controller. Inputs that can be read by the EMS are the driver's accelerator pedal position (APP), brake pedal position (BPP), throttle position (TPS), vehicle speed, engine speed, electric motor speed and battery state-of-charge (SOC). Based on these inputs and a certain control strategy, the EMS gives control commands to the ECU and motor controllers. The driver's demand signal comes from either the throttle position sensor (TPS) or the accelerator pedal position (APP), which is available in vehicles fitted with an electronic throttle. In all conventional vehicles equipped with an ECU, the TPS signal is a significant input for engine operation. Along with other inputs, the TPS signal is used in the ECU control strategy to determine the fuel injection and ignition parameters, which directly affect engine output and performance. Since both the APP and TPS represent the driver's demand for power, either signal can be used as input to determine the power required from the motor. 


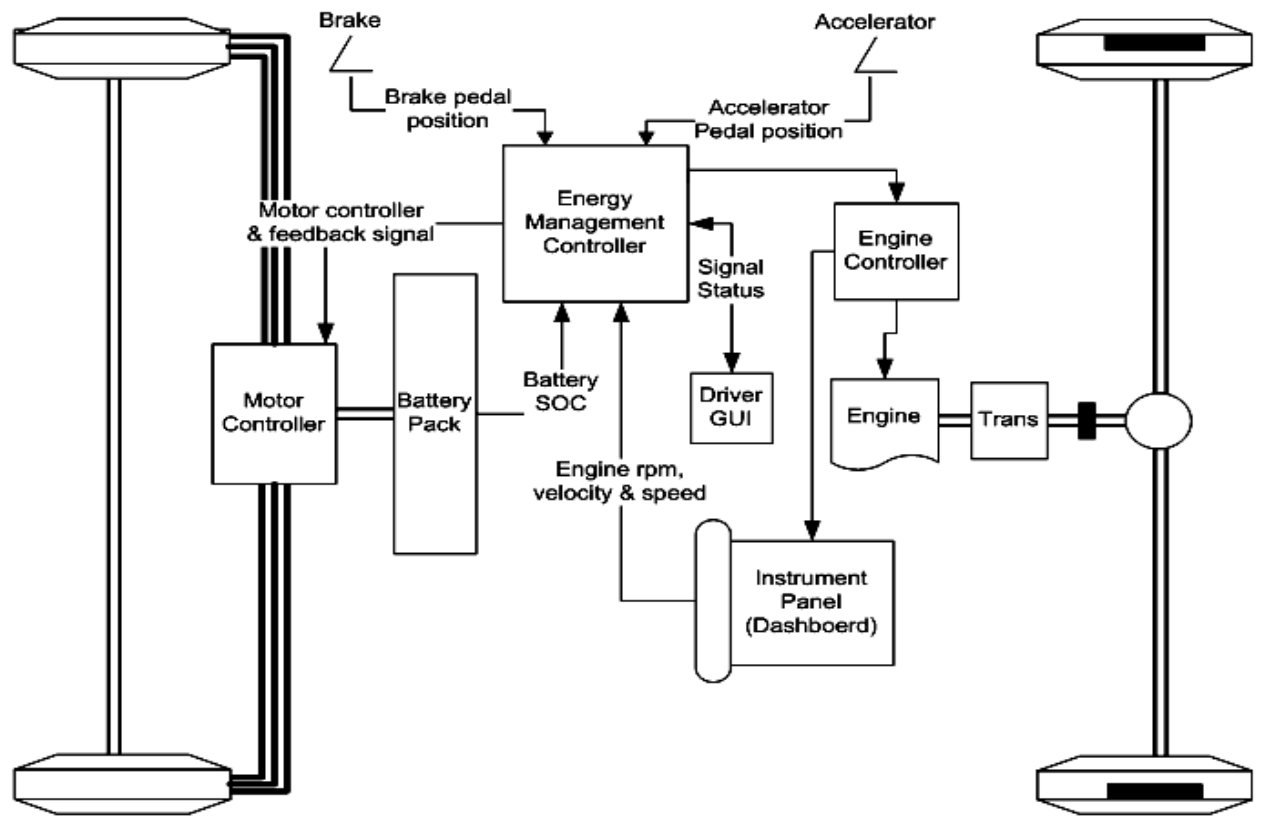

Figure 7. System architecture of split-parallel TTR-IWM hybrid electric vehicle.

The in-wheel motors are driven by dedicated motor controllers which operate in torque control, varying the current fed into the motors; they only perform basic motor commutation to regulate power delivery. The amount of motor energy required is not determined by the existing ECU - a separate EMS controller is needed to carry out the power control strategy, to achieve optimum operation of the hybrid drivetrain. During braking, a brake pedal position (BPP) signal is processed by the EMS, which then instructs the motor controller to reverse the current flow to allow the in-wheel motors to operate as generators to charge the on-board battery bank - a process called re-generative braking, or simply re-gen. The EMS control strategy determines the amount of power to be derived from the generators for battery charging. Finally, there is a graphical driver interface to allow the driver manual override of operating modes, for display of vehicle operating parameters and for in-vehicle data logging.

\section{CONTROL SYSTEM AND CHALLENGES}

\section{Control System for Retrofit TTR-IWM Hybrid Vehicle}

Figure 8 shows control block diagrams and system parameters specific for a retrofit-conversion TTR-IWM hybrid vehicle, highlighting the energy management system (EMS) in blue font, thicker lines and a red-dashed rectangle. In the most basic hybrid conversion possibility (Figure 9), there is no signal going from the EMS to the ECU, with the objective of simplicity and minimal intrusion into existing electronics. As a result, however, operation of the ICE cannot be controlled - the EMS can only operate and control the electric motors. This leads to a serious drawback, since the original look-up maps in the ECU are designed for single-source propulsion only (ICE only) and the pre-tuned engine parameters may not yield optimum performance with the additional power source of the electric motor. In this control scheme, shifting of the ICE operating point cannot be directly controlled. 


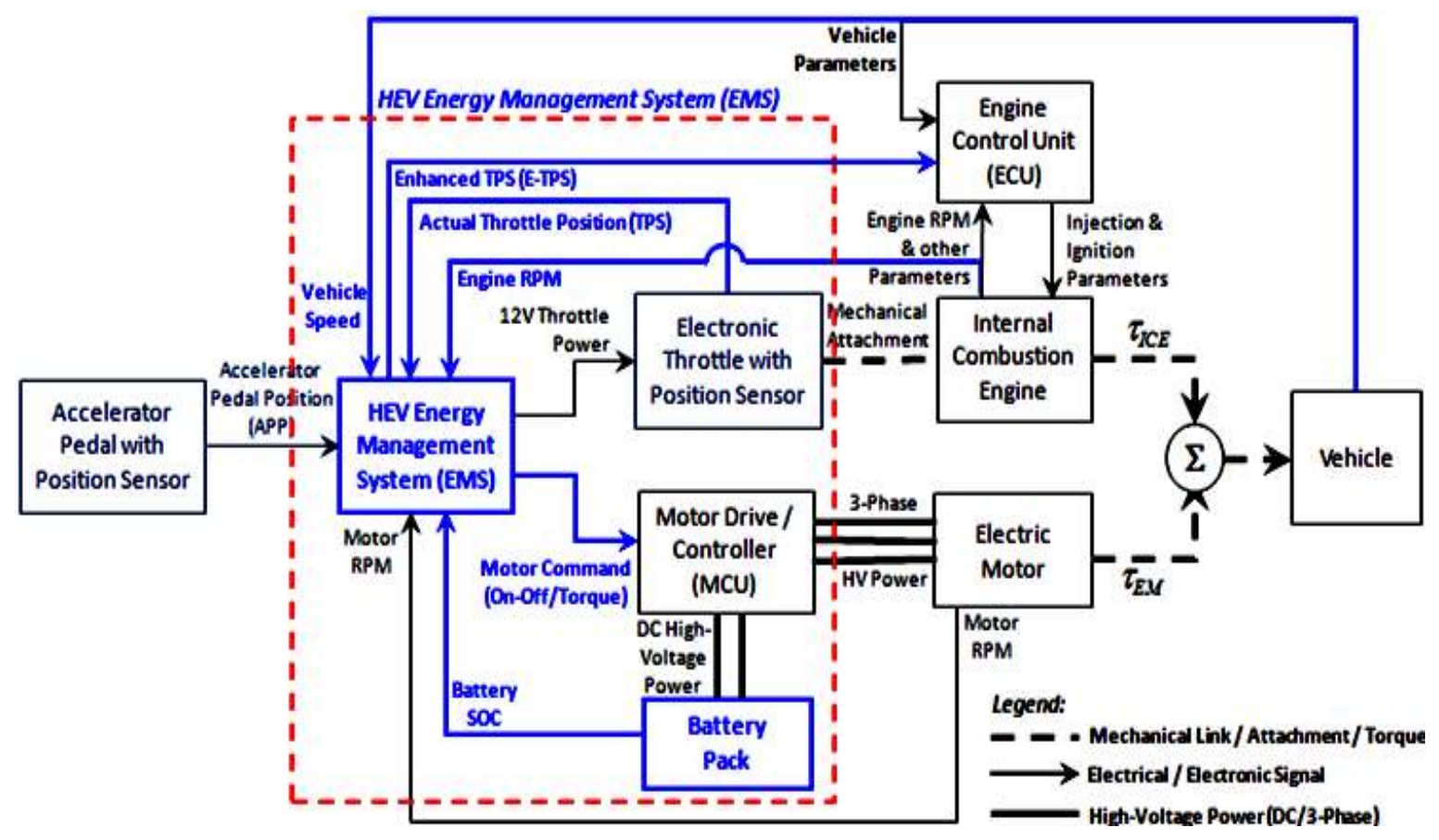

Figure 8. Control block diagram of retrofit TTR-IWM hybrid vehicle: energy management system, with enhanced TPS.
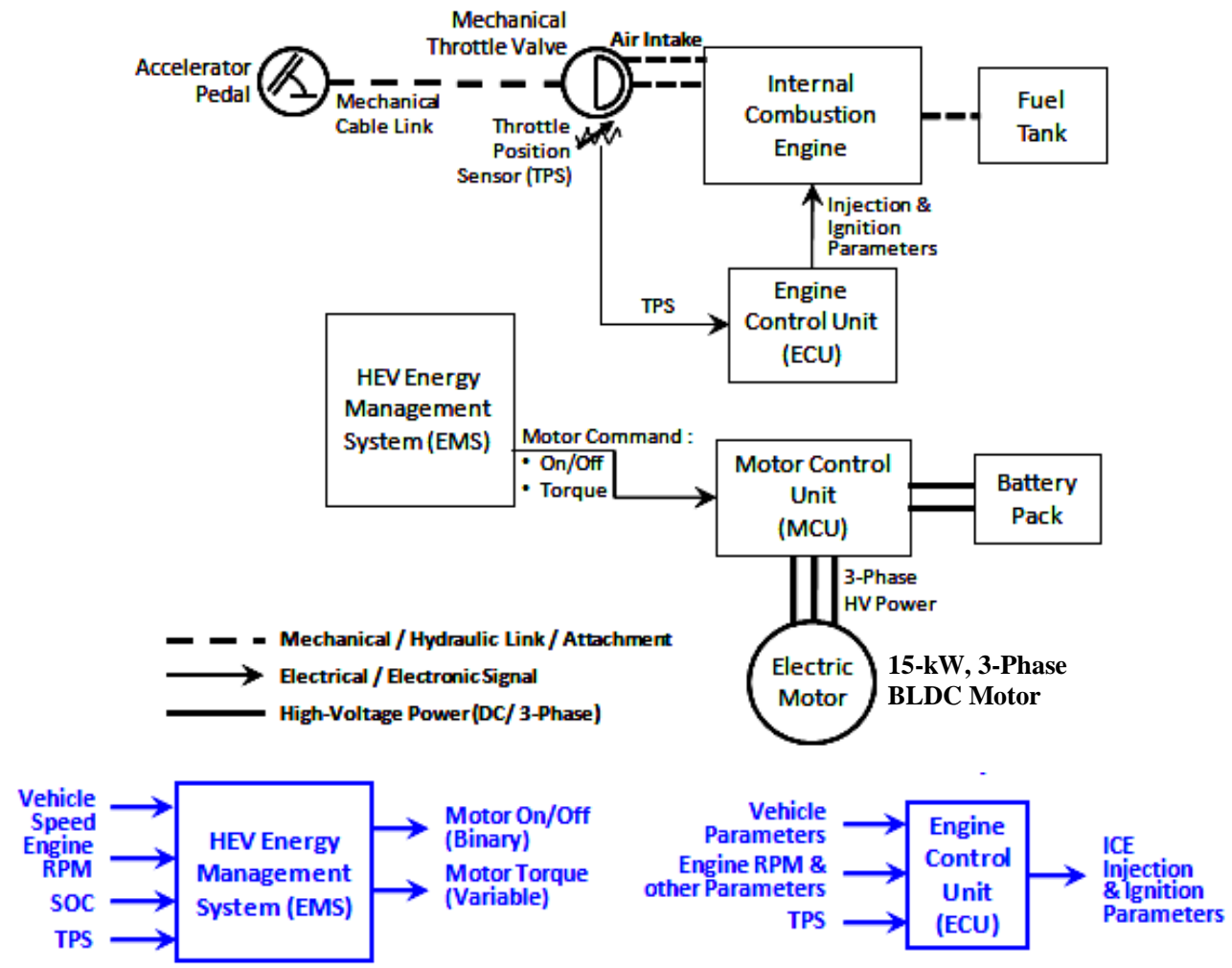

Figure 9. Control system for retrofit TTR-IWM hybrid: basic control (no control of ICE).

Figure 10 shows the implementation of an additional control element, which bypasses the actual TPS signal from the throttle position sensor to the ECU. This will significantly improve control of the hybrid drivetrain since the ICE can be directly 
controlled. This modified or enhanced TPS signal (E-TPS) is generated by the EMS, based on the actual TPS signal from the throttle body and a pre-determined control strategy, to achieve fuel-optimized operation. Altering the TPS signal enables control of the ICE and engine combustion without requiring the vehicle to have an electronic throttle, since the TPS signal is available in all vehicles with ECU. However, since the throttle valve is coupled mechanically to the accelerator pedal, the EMS still cannot control the engine's air intake. In another control possibility (Figure 11), the signal that serves as the driver's demand to the EMS controller is not taken from the TPS, but from the driver's pedal - the accelerator pedal position sensor (APS), which is available in vehicles with a throttle-by-wire system. In these vehicles, the accelerator pedal is not mechanically linked to the throttle valve body, but is just a variable position sensor whose output is an electronic signal read by the ECU, which then uses the signal to vary the throttle opening in closed-loop control and as an input parameter for the engine's combustion system. For the retrofit hybrid conversion, that APS signal is bypassed by the EMS controller, which then produces an enhanced APS signal - based on a control strategy - to be given to the ECU. This, however, is only possible in vehicles already equipped with an electronic throttle system. The difference with the system in Figure 10 is that this configuration controls ICE operation by full control of the throttle system - the EMS has direct control of the throttle and thus engine air intake, as in the case of a purpose-built hybrid vehicle. The retrofit vehicle control systems described above can only achieve the desired objective of hybrid propulsion, namely reduced fuel consumption, with an effective control strategy which determines the optimum power distribution between the ICE and electric motor. Towards this objective, different algorithms should be simulated and analyzed for performance improvement, which is beyond the scope of the present paper.

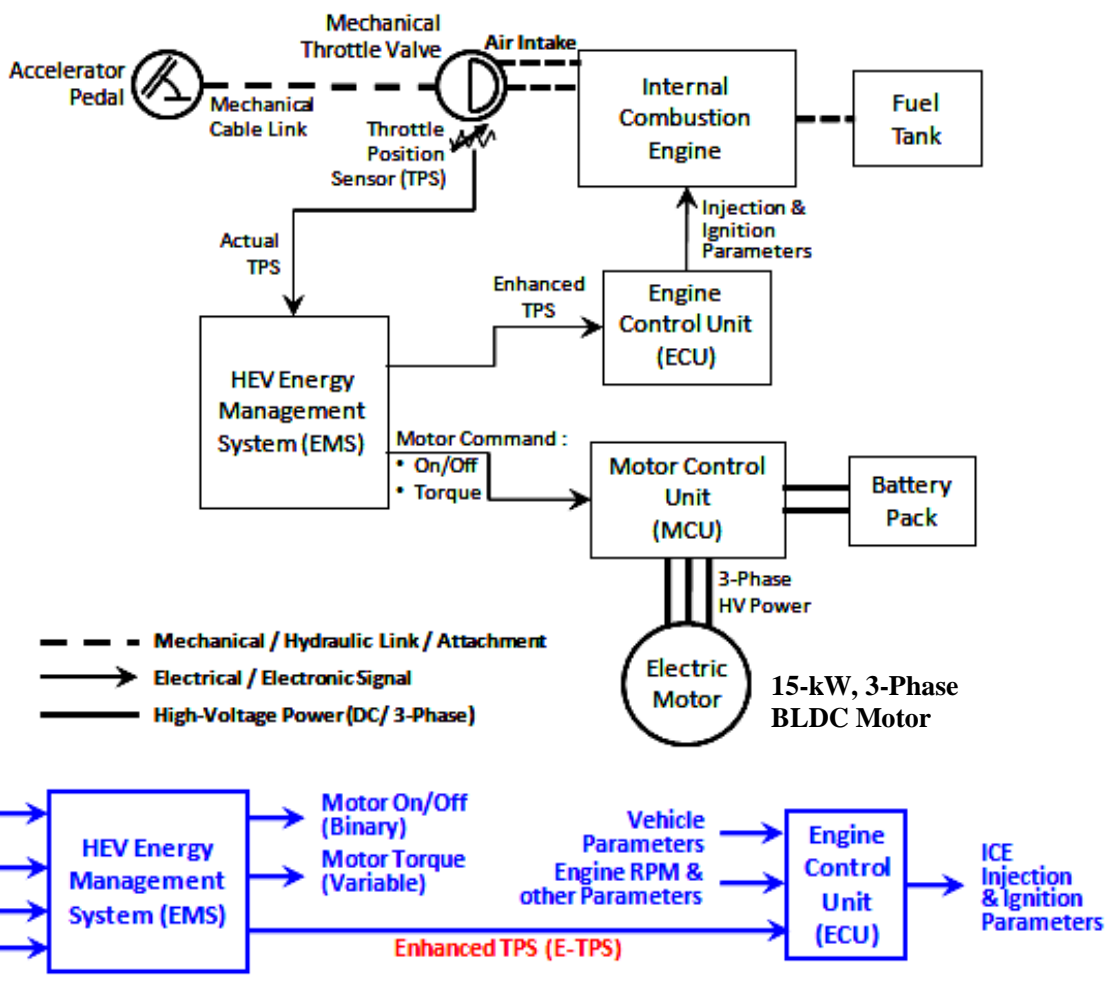

Figure 10. Control system for retrofit TTR-IWM hybrid: enhanced TPS control (no control of air flow/ intake into ICE). 


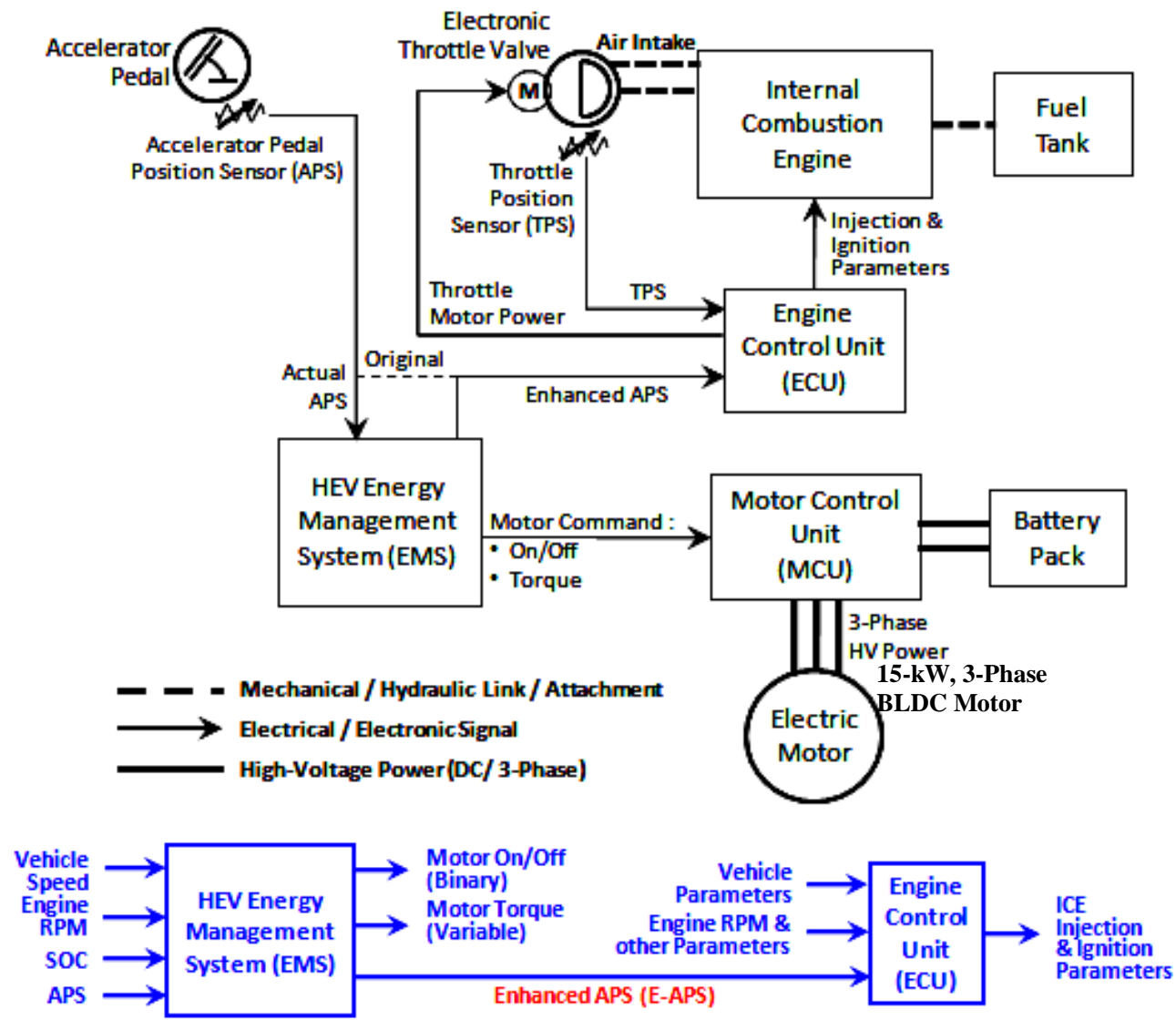

Figure 11. Control system for retrofit TTR-IWM hybrid: enhanced APS control (full control of throttle - only in vehicles with electronic throttle).

\section{Control Challenges of TTR-IWM Hybrid Electric Vehicle}

The main motivation for having a hybrid drivetrain is to reduce the fuel consumption and emissions of the vehicle. Apart from the design and analysis of component sizing, the vehicle's control strategy is a critical factor in ensuring that the operational and control objectives of the hybrid vehicle are achieved. Essentially, the strategy needs to determine the operational status of the two power sources (on/off) and power distribution between them at each point in time, to achieve the following control objectives [9-11]:

1) satisfy the driver's power demand,

2) minimize fuel consumption and emissions,

3) maintain a reasonable level of battery SOC for self-sustaining operation (no external charging), and

4) recover the maximum amount of braking energy.

The hybrid system being considered is for the application of converting a conventional vehicle to a parallel HEV by replacing the rear wheels with in-wheel motors. The disadvantage of the IWM system is that, due to the small design space for the IWM, the maximum torque and power that can be delivered by the motors are rather limited. Thus, the option of using only the motor to provide energy for acceleration from stand-still and during low vehicle speeds is not available in the TTR-IWM hybrid - a drawback of the IWM-retrofit system, since fuel and emissions reduction is significant during acceleration and low-speed operation. Another disadvantage is that battery 
charging in the TTR-IWM hybrid can only occur when the vehicle is moving, thereby reducing the charging opportunity of the vehicle [12]. These two factors are the primary challenges for operation and control of the TTR-IWM hybrid, leading to significant differences from the chassis-mounted hybrid system. These are shown in Table 1, which further categorizes the operating modes of the TTR-IWM hybrid, according to different driving conditions. In a retrofitted hybrid, the vehicle maintains its original engine and system components. Since the engine is not down-sized, the converted vehicle's acceleration and gradeability are not significantly downgraded from the original vehicle, since the difference is only in the increase of vehicle mass, due to the added motors and batteries. This is shown in Zulkifli, Mohd [8], which investigates the influence of motor size and power on a retrofitted vehicle's performance, for different motor ratings and vehicle sizes.

In a purpose-built parallel hybrid vehicle, the control strategy that is most widely used is the electric assist strategy. The main power source for the vehicle is the ICE, while the electric motor-generator acts as a power buffer to compensate for the difference in the power requirement - running it as a motor to make up for a lack of propulsion power, or as a generator to absorb additional power to charge the batteries. The improved fuel performance arises from shifting the ICE's operating point closer to the optimal regions of fuel economy and efficiency, at each engine speed. At the same time, battery SOC is maintained by a charge-sustaining strategy which regulates the SOC within pre-defined levels [13-16]. When the vehicle moves from a stand-still, the engine operates with low efficiency. In a hybrid vehicle with an on-board motor, the vehicle runs in full-electric mode, to reduce fuel consumption and emissions. In a TTR-IWM hybrid, the insufficient in-wheel motor torque demands a hybrid operation. However, with less torque required from the engine due to the available motor torque, this may result in an even less efficient ICE operating point. It may be better to delay turning on the motor. Thus, considerations for acceleration from stand-still include: 1) operating only the ICE - which may result in low engine efficiency and more emissions, 2) hybrid operation from the start - which may still give low engine efficiency while emissions improvement is unclear, and 3) delayed hybrid operation - which may result in more fuel and emissions when only the ICE is propelling $[1,2,7]$.

To determine the optimal operation, simulation tests are necessary to investigate the effect of delaying the motor start time, for different rates of vehicle acceleration [17, 18]. When the battery SoC is below the minimum threshold, the charging mode is effective. However, the generator cannot operate when the vehicle is stationary. As stated in Table I, to make up for the reduced charging opportunity, extra torque must be requested from the ICE when the vehicle is moving, to provide charging power for the battery pack (in addition to the required propulsion torque). Whenever the battery is charged by the ICE (not from re-gen braking), fuel is actually consumed (conversion from chemical to mechanical and electrical energy). In an ordinary parallel HEV, charging during vehicle stand-still entails low-efficiency ICE operation. In the TTR hybrid, it is thus better that battery charging is performed when the vehicle is in motion because the ICE operating point can be shifted to a higher efficiency region (due to the existing propulsion torque). This is investigated through dynamic simulation and analysis, which is reported in a different work by the same authors. 
Table 1. Comparison of operating modes between ordinary parallel and TTR-IWM parallel hybrid electric vehicles.

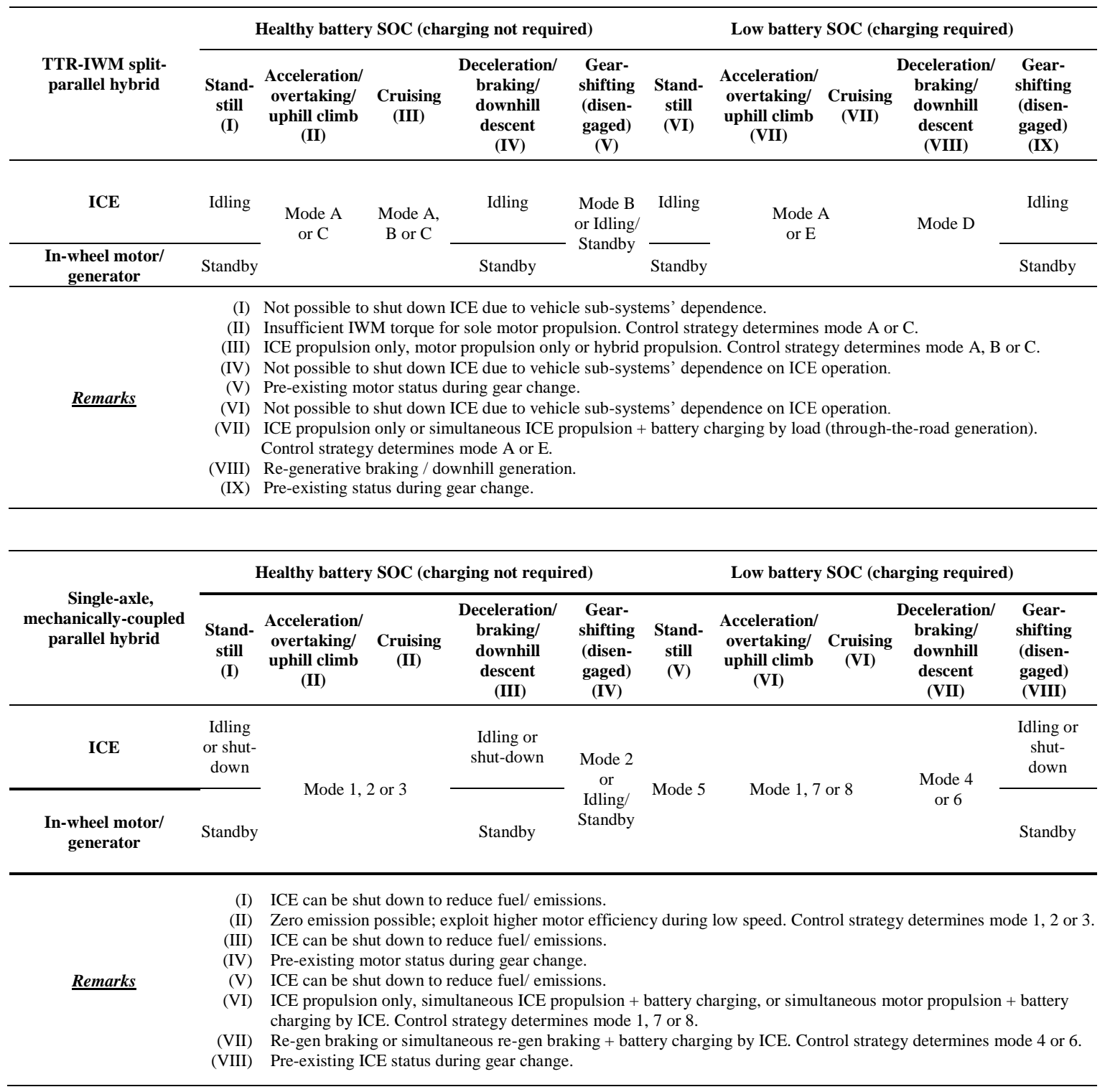

\section{SIMULATION RESULTS OF ACCELERATION}

Initial simulation results are given below, comparing the acceleration performance of the TTR-IWM hybrid vehicle and the more common parallel hybrid configuration: the pre-transmission, device-coupled hybrid. Acceleration tests are carried out on a small vehicle whose specifications are given in [8] for three different motor sizes $(16-\mathrm{kW}$, $32-\mathrm{kW}$ and $75-\mathrm{kW}$ continuous power). Figure 12 shows that with each motor, the acceleration performance improves from the conventional vehicle, and is always better (faster) than the pre-transmission hybrid, with the AC75 hybrid showing the biggest difference and the PM16 hybrid having the fastest response. The same patterns are observed in fuel consumption (Figure 13). However, for emissions (Figure 14), all TTR-IWM hybrids show increased levels from the pre-transmission hybrids, for all emission types (the biggest difference in the AC75 hybrid). 

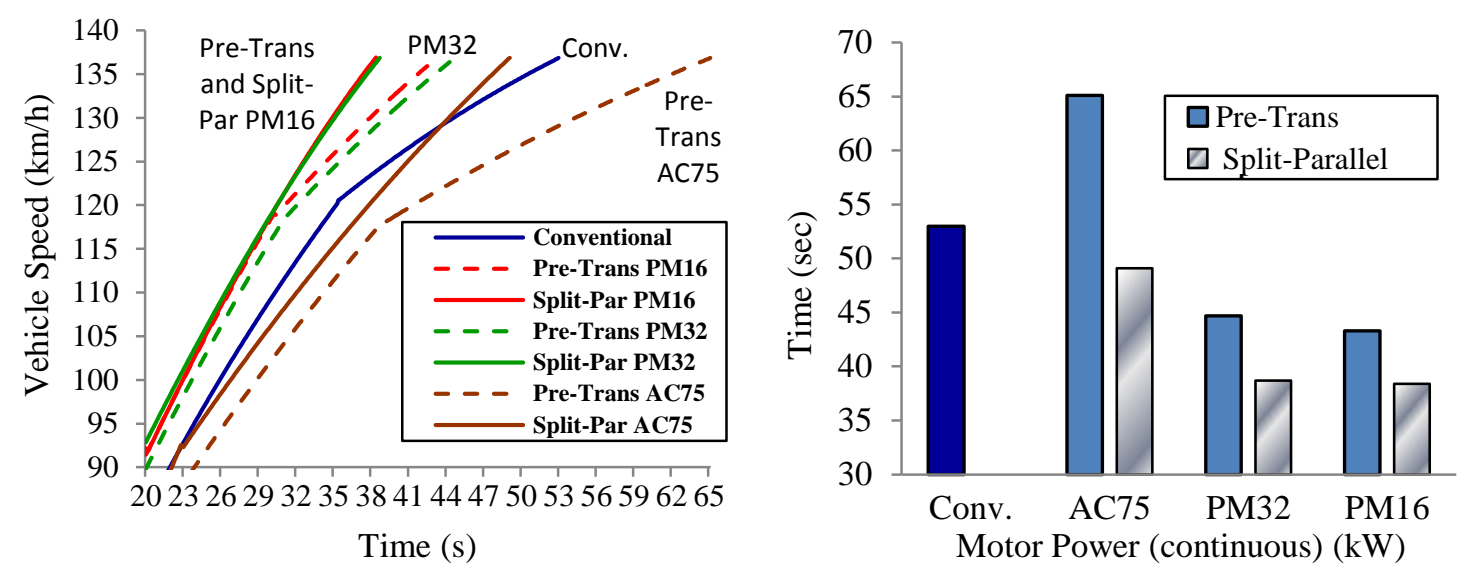

Figure 12. Vehicle speed and acceleration performance.

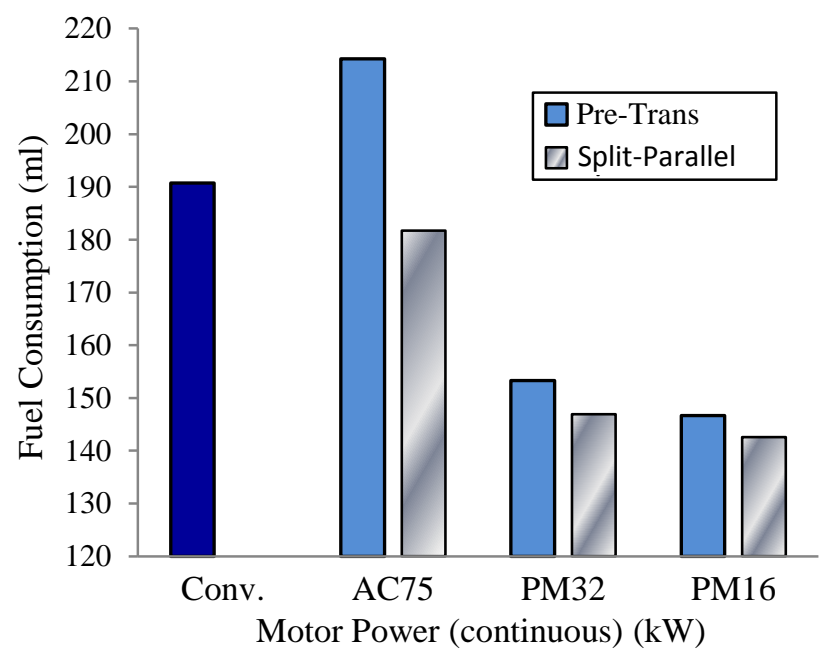

Figure 13. Fuel consumption.
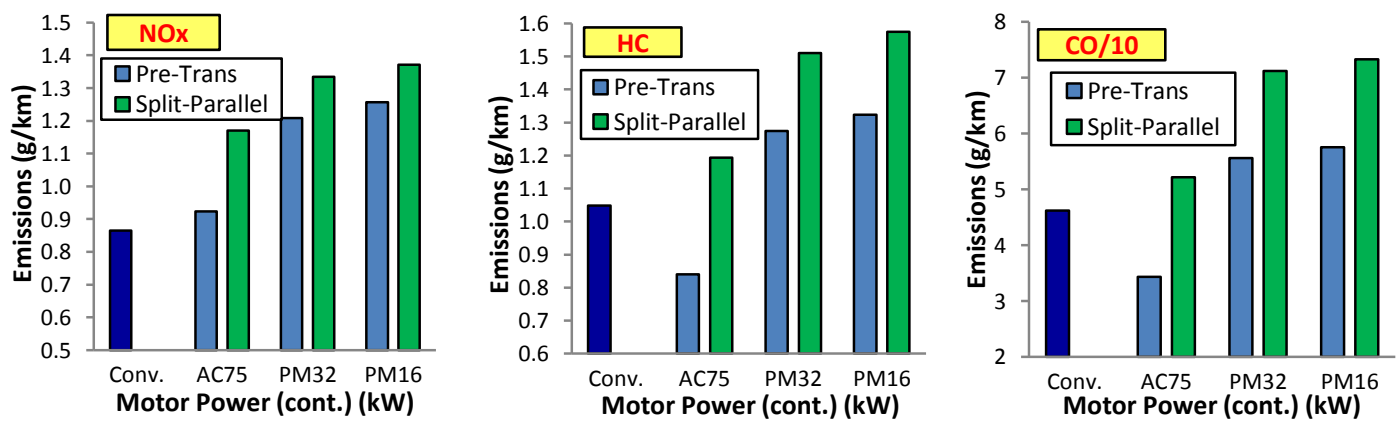

Figure 14. Emissions - $\mathrm{NO}_{\mathrm{x}}, \mathrm{HC}$ and $\mathrm{CO}$.

\section{CONCLUSIONS}

A split-axle parallel hybrid configuration enables existing ICE-powered vehicles to be converted into hybrid electric vehicles with minimal physical modification, to be a through-the-road hybrid with in-wheel motors (TTR-IWM). This paper describes the specific operating modes and power flow of the TTR-IWM hybrid, along with the drivetrain configuration, torque-speed profile and system architecture. A discussion of 
control objectives, control system and parameters and specific control challenges in a retrofit-conversion TTR-IWM hybrid vehicle is also provided. Simulation results show that the TTR-IWM hybrid has better fuel economy and acceleration performance, compared to the pre-transmission, parallel hybrid electric vehicle with on-board motor.

\section{ACKNOWLEDGEMENTS}

This work is partly funded by an ERGS grant (Exploratory Research Grant Scheme) from the Ministry of Higher Education, Malaysia.

\section{REFERENCES}

[1] Rahmat MS, Ahmad F, Mat Yamin AK, Aparow VR, Tamaldin N. Modeling and torque tracking control of permanent magnet synchronous motor (PMSM) for hybrid electric vehicle. International Journal of Automotive and Mechanical Engineering. 2013;7:955-67.

[2] Salleh I, Md. Zain MZ, Raja Hamzah RI. Evaluation of annoyance and suitability of a back-up warning sound for electric vehicles. International Journal of Automotive and Mechanical Engineering. 2013;8:1267-77.

[3] Zulkifli S, Saad N, Mohd S. Split-parallel in-wheel-motor retrofit hybrid electric vehicle. IEEE International Power Engineering and Optimization Conference; 2012: 11-6.

[4] Khayyam H, Kouzani A, Nahavandi S, Marano V, Rizzoni G. Intelligent energy management in hybrid electric vehicles, in Energy Management: Vukovar, Croatia: InTech; 2010.

[5] Ehsani M, Gao Y, Emadi A. Modern electric, hybrid electric, and fuel cell vehicles: fundamentals, theory, and design. CRC press; 2009.

[6] Chan C-C, Bouscayrol A, Chen K. Electric, hybrid, and fuel-cell vehicles: Architectures and modeling. IEEE Transactions on Vehicular Technology. 2010;59:589-98.

[7] Bakhtiari-Nejad F, Nazemizadeh M, Arjmand H. Tracking control of an underactuated gantry crane using an optimal feedback controller. International Journal of Automotive and Mechanical Engineering. 2013;7:830-9.

[8] Zulkifli SA, Mohd S, Saad NB, Aziz ARA. Impact of motor size \& efficiency on acceleration, fuel consumption \& emissions of split-axle through-the-road parallel hybrid electric vehicle. Applied Mechanics and Materials. 2014; 663: 498-503.

[9] Young M, Molen GM, Oglesby D, Crawford K, Walp K, Lewis R, et al. The design and development of a through-the-road parallel diesel electric hybrid. IEEE Vehicle Power and Propulsion Conference; 2007: 511-8.

[10] Fallahi N, Niasar AH. Intelligent energy management strategy for a separated-axle parallel hybrid electric vehicle. Intelligent Systems in Electrical Engineering. 2014;4: 51-61.

[11] Asaei B, Habibidoost M. A new energy control strategy for a through the road parallel hybrid electric motorcycle. IEEE Vehicle Power and Propulsion Conference; 2010: 1-5.

[12] Sheu K, Hsu T. Design and implementation of a novel hybrid-electric motorcycle transmission. Applied Energy. 2006;83:959-74. 
[13] Sciarretta A, Back M, Guzzella L. Optimal control of parallel hybrid electric vehicles. IEEE Trans on Control Systems Technology. 2004;12:352-63.

[14] Lin C, Peng H, Grizzle J, Kang J. Power management strategy for a parallel hybrid electric truck. IEEE Transactions on Control Systems Technology. 2003;11:839-49.

[15] Schouten N, Salman M, Kheir N. Fuzzy logic control for parallel hybrid vehicles. IEEE Transactions on Control Systems Technology. 2002;10:460-8.

[16] Baumann B, Washington G, Glenn B, Rizzoni G. Mechatronic design and control of hybrid electric vehicles. IEEE/ASME Transactions on Mechatronics. 2000;5:58-72.

[17] Zulkarnain N, Zamzuri H, Mazlan SA. Ride and handling analysis for an active anti-roll bar: case study on composite nonlinear control strategy. International Journal of Automotive and Mechanical Engineering. 2014;10:2122-242.

[18] Nagarkar MP, Vikhe GJ, Borole KR, Nandedkar VM. Active control of quarter car suspension system using linear quadratic regulator. International Journal of Automotive and Mechanical Engineering. 2011;3:364-72. 